\title{
Les matériaux élastomériques
}

Aurélie PIC ${ }^{*}$

75 avenue Henri Delecaux, 59130 Lambersart, France

\section{Composition, structure macromoléculaire et dynamique}

\subsection{Origines et composition}

Les élastomères sont des polymères de haute masse moléculaire et à chaînes quasiment linéaires. Les macromolécules (ensembles de monomères), entrelacées comme un tas de vers particulièrement longs, forment occasionnellement des ponts entre elles. Le nombre de ponts doit rester faible, environ un pont pour quelques centaines de monomères. Sous contrainte, les molécules glissent par reptation sauf au niveau des ponts. Ces derniers donnent au matériau une mémoire de forme : qu'on lui appose une contrainte mécanique d'élongation et les segments entre deux ponts s'étirent alors; qu'on supprime l'élongation, et les mouvements de relaxation entre les molécules les ramènent à leur configuration, et donc à leur forme initiale. La contrainte tend à ordonner les molécules du matériau; la suppression de la contrainte lui permet de retrouver le désordre originel [3].

Un élastomère est donc un polymère dit «élastique »: il supporte de très grandes déformations ( $\gg 100 \%$ ), presque totalement réversibles.

Le terme élastomère désigne ainsi tous les caoutchoucs synthétiques, c'est-à-dire les substances macromoléculaires possédant l'élasticité dite caoutchoutique [51].

En effet, le caoutchouc naturel a été le premier élastomère connu, il est obtenu à partir du latex, un liquide blanc ou jaune sécrété par certains végétaux dont le pissenlit et surtout l'hevea brasiliensis, végétal de la famille des euphorbiacées. La solution de latex donne environ 36 à $40 \%$ d'extrait sec dont $92 \%$ de caoutchouc [40]. La formule du caoutchouc

*Auteur pour correspondance : picaurelie@yahoo.fr naturel est le cis 1,4 polyisoprène. Il a été introduit en France par La Condamine au XVIII ${ }^{\mathrm{e}}$ siècle, mais son emploi est longtemps limité du fait de sa sensibilité à la chaleur et de ses propriétés hydrophiles. En 1839, Charles Goodyear donne au caoutchouc de nouvelles propriétés grâce au procédé de vulcanisation.

C'est à partir de cette période que l'utilisation d'élastiques (bracelets en caoutchouc) a commencé à se développer en orthodontie. Baker publie un article en 1846, il a surtout employé le caoutchouc comme ligature. Tomes les utilise en 1847, puis de nombreux auteurs en vantent les avantages: Tucker qui recommande d'acheter des anneaux en caoutchouc et de "les couper transversalement ", Kingsley en 1866, Kuehns, Case. Angle décrit l'emploi des élastiques intermaxillaires verticaux en 1891; Grandhomme dit les utiliser depuis 1933 [43]. .

Au début du $20^{\mathrm{e}}$ siècle, les chimistes prennent le relais des explorateurs et parviennent à synthétiser le caoutchouc à partir du pétrole. Le raffinement industriel permet d'introduire en orthodontie l'usage des premières chaînettes élastomériques dans les années soixante. Elles sont dès lors devenues partie intégrante de nombreux traitements [12].

Les élastomères synthétiques sont des polymères amorphes de matériaux polyuréthane.

Les élastomères à base de polyuréthane sont fabriqués suivant plusieurs étapes [11] :

- un intermédiaire de base est d'abord utilisé sous forme d'un polymère de masse moléculaire basse, souvent un polyester ou un polyéther;

- l'intermédiaire réagit ensuite avec un diisocyanate aromatique pour aboutir à un prépolymère;

- cet élastomère est ensuite vulcanisé par réaction des groupements isocyanates avec des alcools ou des glycols. 


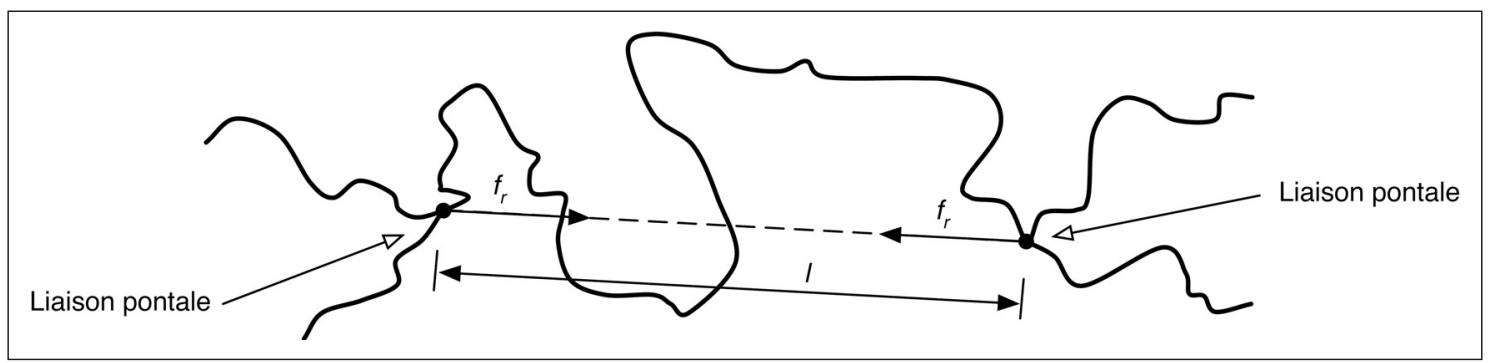

Figure 1

Force de rétraction élastique entre les extrémités de la chaîne d'un élastomère (d'après Mercier, et al. [37]).

La vulcanisation est un traitement par le soufre et la chaleur qui permet d'améliorer les propriétés en provoquant l'établissement de liaisons intermoléculaires qui augmentent la stabilité du caoutchouc tout en lui conservant ses propriétés d'élasticité.

Pour donner forme à leurs produits, les compagnies de matériaux orthodontiques utilisent ensuite l'un ou l'autre de ces deux types de processus de fabrication [11] :

- le moulage par injection,

- le matriçage « die stamping », qui confère au matériau des qualités physiques plus constantes.

La structure élastomérique du produit fini diffère selon les maisons dentaires. Par conséquent, leurs propriétés et les forces qu'ils délivrent varient également [44].

Peu de données sont disponibles sur le processus de pigmentation; si les pigments peuvent former des ponts avec le polymère alors il existe un risque potentiel de modifier les propriétés des élastomères [11].

En comparant les élastomères colorés de trois marques différentes, Renick n'a mis en évidence une modification de la température de transition vitreuse que pour l'une d'entre elles [44].

Les élastomères contiennent de 50 à $60 \%$ de polymères. Le reste est composé de charges, agents de vulcanisation, accélérateurs, produits retardant le vieillissement et d'autres additifs qui permettent de modifier la matière première, afin de répondre aux exigences d'une application définie.

\subsection{Structure moléculaire et dynamique}

Un élastomère est constitué de longues chaînes moléculaires rassemblées, au repos, en «pelotes» (Fig. 1). Ces chaînes sont reliées entre elles par des points de réticulation, des enchevêtrements ou encore des liaisons polaires avec des charges minérales. Elles forment un réseau.

Les propriétés mécaniques des élastomères dépendent en premier lieu de la longueur des chaînes entre les nœuds du réseau. Plus cette longueur est faible (réseau dense) plus l'élastomère est rigide.

En extension ou en torsion du matériau, les chaînes moléculaires se déplient et adoptent un ordre linéaire qui est réalisé aux dépens des liaisons secondaires, les liaisons primaires restant effectives. Larrêt des contraintes d'extension-torsion permet donc aux chaînes moléculaires de retrouver leur configuration passive, repliée et aléatoire (déformation élastique), à condition que les contraintes n'aient pas rompu les liaisons primaires, auquel cas la déformation devient irréversible (passage dans le domaine plastique) [37].

\section{Propriétés mécaniques}

\subsection{Déformation et élasticité}

Toute force appliquée à un matériau se traduit par une déformation. Cette déformation à l'échelle microscopique entraîne l'apparition d'une force de rétraction qui tend à restituer à l'échantillon sa forme originale. Si la force de rétraction est constante dans le temps, le matériau a un comportement élastique [37].

Presque tous les matériaux ont une réponse élastique mais seulement pour de petites déformations (environ $0,1 \%$ ) et cette déformation ( $\boldsymbol{\varepsilon}$ ) est proportionnelle à la contrainte $\sigma$ appliquée (loi de Hooke) : $\sigma=\mathrm{E} \varepsilon$. La loi de Hooke décrit que, pour de petites déformations, la déformation $(\varepsilon)$ est quasiment proportionnelle à la contrainte appliquée dans de nombreux matériaux ( $\mathrm{E}=$ module de Young). Au-delà, 


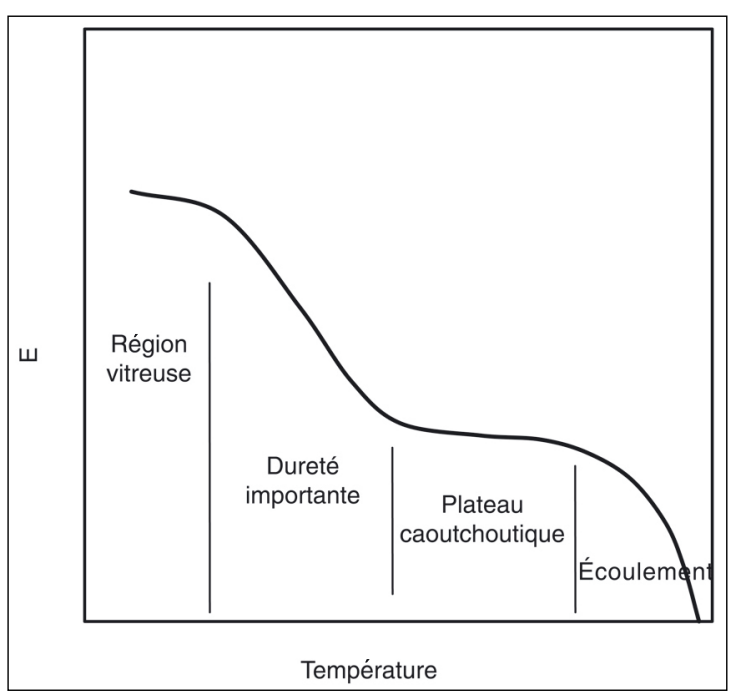

Figure 2

Variation du module d'élasticité des polymères en fonction de la température (d'après Brantley, Eliades [11]).

ils subissent un phénomène irréversible : la plupart d'entre eux gardent une déformation plastique qui change leur forme de manière permanente. Les élastomères présentent un faible module d'élasticité ou module de Young : 0,01 à $0,1 \mathrm{GPa}$ (diamant : $1000 \mathrm{GPa})$.

La rigidité des polymères varie en fonction des forces intermoléculaires entre les chaînes. Le module d'élasticité des polymères dépend également de la température [16] (Fig. 2).

Ce qui rend les élastomères particuliers, c'est leur capacité de collisions élastiques (conservation de l'énergie cinétique totale des corps qui se heurtent, et conservation de la quantité de mouvement si absence de frottement) tels le rebond et l'étirement élastique, qui leur permet de reprendre leur forme initiale après étirement. Cette caractéristique spéciale est acquise par l'enchevêtrement de longues chaînes polymériques.

Les propriétés des élastomères dépendent de leur degré de réticulation; c'est ainsi que les propriétés du caoutchouc varient en fonction de la quantité de soufre utilisée lors de la vulcanisation, en vue de lier les chaînes les unes aux autres [16].

\subsection{Allongement et module de rigidité}

Les élastomères subissent une transition vitreuse en dessous de la température ambiante $\left(\mathrm{de}-50^{\circ} \mathrm{C}\right.$ à $-80{ }^{\circ} \mathrm{C}$ pour les polyuréthanes). En dessous de la température de transition vitreuse, ils sont relativement rigides, alors qu'au-dessus de cette température, ces matériaux deviennent hautement flexibles avec une activation thermique. Plus la température de transition vitreuse est élevée, plus le polymère est rigide, et la formation d'un polymère plus rigide est associée avec une force délivrée plus importante, ou en d'autres termes, avec un module élastique plus élevé. Ils contiennent aussi très souvent une seconde phase de polymère cristallin qui subit une fusion au dessus de la température de transition vitreuse [11].

La déformation qui résulte de l'application d'une force de traction est l'allongement. Lallongement de l'élastomère est instantané sous l'effet d'une tension et atteint des proportions importantes en relation avec un faible module de rigidité initial. Ils sont capables de s'allonger élastiquement de $300 \%$ ou même plus, allongement qui disparaît à la décharge. Cependant, l'allongement devient de plus en plus difficile au fur et à mesure que la contrainte augmente d'intensité. Le module de rigidité, initialement faible, augmente pour atteindre son maximum au moment de la rupture de l'échantillon. Lélastomère étiré, observé aux rayons X, présente un arrangement spécifique comparable à un état cristallin et c'est cette cristallisation du matériau qui entraînerait un renforcement et l'augmentation du module de rigidité [32].

La rigidité du réseau dépend de la longueur et de la nature chimique des chaînes polyols entre les ponts "uréthanes ». Pour des chaînes de même nature chimique, plus les chaînes sont courtes, plus les ponts sont rapprochés, et plus le système est rigide [3].

A contrario, les élastomères sont des matériaux incompressibles (ou très peu compressibles) : coefficient de Poisson de 0,5.

\subsection{Force de rupture}

Elle correspond à la force qui va provoquer la rupture de l'élément élastique. Lallongement de rupture est de l'ordre de $1000 \%$ et peut être porté à 10000 \% après quelques étirements - relâchements. Ce phénomène appelé «rackage » est dû à l'apparition d'une structure fibreuse au sein de l'élastomère. En orthodontie, cette notion est peu retenue, 


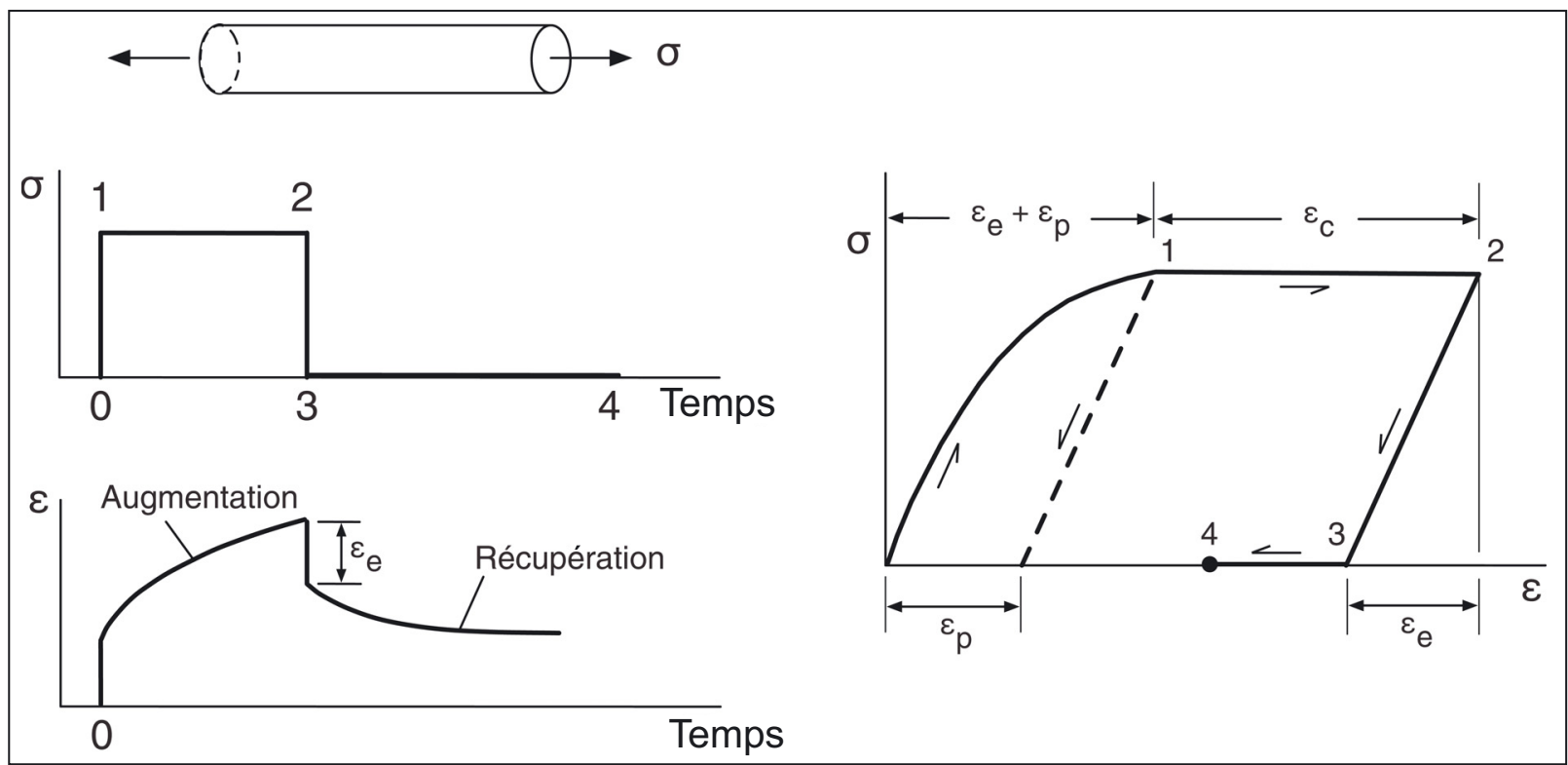

Figure 3

Charge et décharge d'un matériau viscoélastique. La déformation élastique initiale instantanée correspond à 1, et la région entre 1 et 2 est une combinaison de la déformation retardée et de la déformation permanente. La première portion de la courbe de décharge entre 2 et 3 est la récupération de la déformation élastique instantanée. La déformation plastique permanente est désignée en 4. (D’après Brantley, Eliades [11].)

car l'étirement initial des chaînettes est très en deçà de ce seuil de rupture [1].

Les élastomères sont donc caractérisés par une grande résistance mécanique : capacité élevée à éviter l'effondrement par déformation plastique ou par rupture.

Le caoutchouc naturel présente une faible résistance à la traction (Rm) : $30 \mathrm{MPa}$ (acier : 760-1280 MPa).

\subsection{Viscoélasticité : comportement élastique non linéaire}

D'autres comportements existent où le domaine élastique peut ne pas être linéaire. C'est le cas des élastomères ou caoutchoucs pour lesquels charge et contrainte ne restent pas proportionnelles. Les chaînes de polymères sont d'abord, sous l'effet de la contrainte, alignées, ce qui donne une appréciable déformation élastique sous faible contrainte. Ensuite, les chaînes sont étirées, toujours dans le domaine élastique, et les distances inter-atomiques allongées: c'est la déformation élastique retardée ou déformation viscoélastique $[11,16]$. L'effet global conduit à une élasticité non linéaire (Fig. 3). Si la contrainte se poursuit, une déformation plastique apparaît. Le matériau est toujours élastique : en relâchant la contrainte, on décrit la même courbe que lorsque la courbe croissait, et toute l'énergie stockée pendant la mise en charge est récupérée à la décharge [12].

Les modules élastomériques composés d'uréthane présentent une élasticité dite caoutchoutique : la courbe charge / allongement n'est pas linéaire (comme pour les métaux et les céramiques) mais incurvée. La limite élastique se situe au changement d'inflexion [11]. La déformation élastique n'est donc pas proportionnelle à la charge qui la provoque (Fig. 4). La proportionnalité entre la contrainte et la déformation n'est pas constante, comme l'exprime la loi de Hooke. Lorsque la contrainte est nulle, ces matériaux sont amorphes; leurs chaînes tendant toutefois à s'aligner au cours de la déformation, il se produit une augmentation de la rigidité [16].

Létude de comportement viscoélastique nécessite de préciser la durée d'application de la contrainte [16]. De plus, l'environnement des polymères est un paramètre très important dans leur comportement à long terme et en particulier la présence d'eau, d'électrolytes ou d'autres composés [26].

La vitesse de la contrainte joue également un rôle : il est recommandé d'activer doucement in vivo 


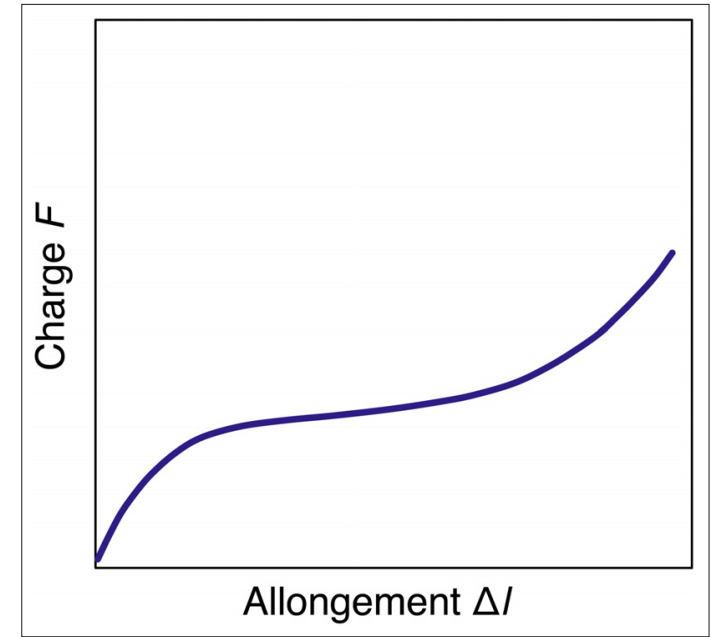

Figure 4

Courbe charge/allongement (d'après Ebaïlon, Dorlot [16]).

pour obtenir un minimum de dégradation de la force [11].

\subsection{Mémoire évanescente}

Dans les matériaux viscoélastiques, les déformations déterminées au moment de référence dépendent de toute l'histoire des contraintes mécaniques subies par le matériau auparavant. Ce type de matériau est appelé "matériau à mémoire évanescente », car il garde en mémoire les déformations subies au cours de sa fabrication et durant son utilisation mais l'effet de ces contraintes s'estompe au cours du temps. C'est pourquoi certains auteurs recommandent de pré-étirer les chaînettes [32].

\section{Vieillissement et biodégradation}

\subsection{Biodégradation fonctionnelle}

Les composants élastomériques sont incapables d'exercer une force d'intensité constante pendant une longue période.

Ceci est en partie lié au comportement viscoélastique.

En élasticité, la relation entre contrainte et déformation est indépendante du mode d'application de la contrainte ou de la déformation. En viscoélasticité, ce n'est pas le cas et il faut préciser le protocole expérimental utilisé pour appliquer la contrainte ou la déformation.
On définit ainsi deux modes de déformation : la relaxation et le fluage.

On appelle fluage d'un matériau, le phénomène de déformation irréversible qui augmente avec le temps sous l'effet d'une contrainte constante (Fig. 5).

Dans une expérience de fluage, on impose un échelon de contrainte et on évalue la déformation au cours du temps.

La vitesse de fluage augmente généralement avec la température du matériau. La déformation par fluage peut se modéliser comme un frottement fluide, du type amortisseur de suspension de voiture. De la même manière que pour la déformation plastique, le fluage est toujours associé à de la déformation élastique. On peut voir le fluage comme un «retard à la déformation »: si l'on impose une déformation, on a d'abord une réponse élastique, puis la force diminue bien que la déformation soit maintenue constante.

La relaxation est la diminution de la force pour un allongement constant, elle est due à différentes déformations engendrées par l'allongement de l'élastomère.

La relaxation consiste à imposer au matériau un échelon de déformation, et à observer l'évolution de la contrainte au cours du temps (Fig. 6).

Comme tout matériau viscoélastique, l'élastomère est très dépendant de la vitesse de déformation à laquelle on le soumet.

Les déplacements dentaires ne doivent pas être négligés: plus la vitesse de déplacement augmente, plus la déperdition de force augmente également [1]

\subsection{Biodégradation environnementale}

Toutes les études montrent que la déperdition de force est plus importante in vivo que in vitro $[6,32]$.

La dégradation des propriétés physiques et chimiques au sein du milieu humide et agressif que constitue la cavité buccale est un facteur d'aggravation du vieillissement et de la porosité des élastomères.

En bouche, différents facteurs vont influer :

- facteurs chimiques: salive (enzymes, flore bactérienne complexe), nourriture, hygiène,

- facteurs thermiques: variations de température des aliments,

- variations de $\mathrm{pH}$,

- facteurs mécaniques : mastication, brossage. 


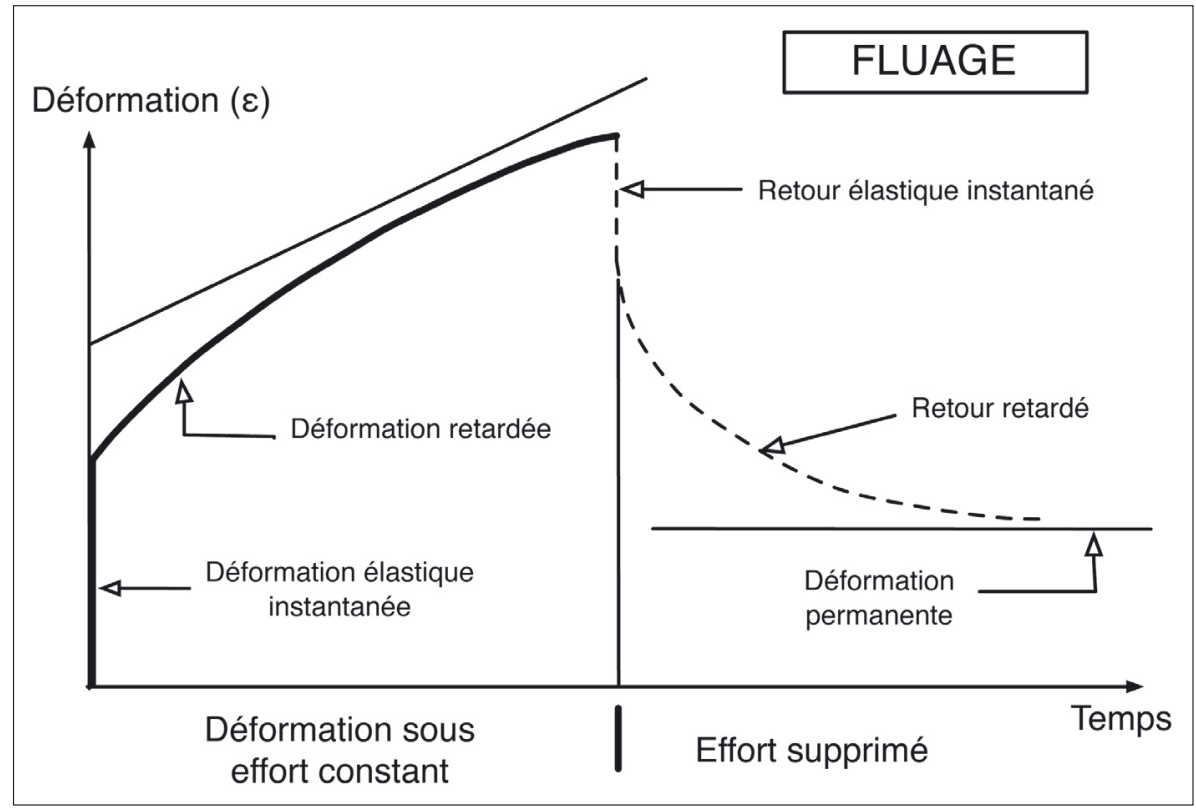

Figure 5

Expression du fluage d'un matériau visco-élastique (d'après Montandreau [39]).
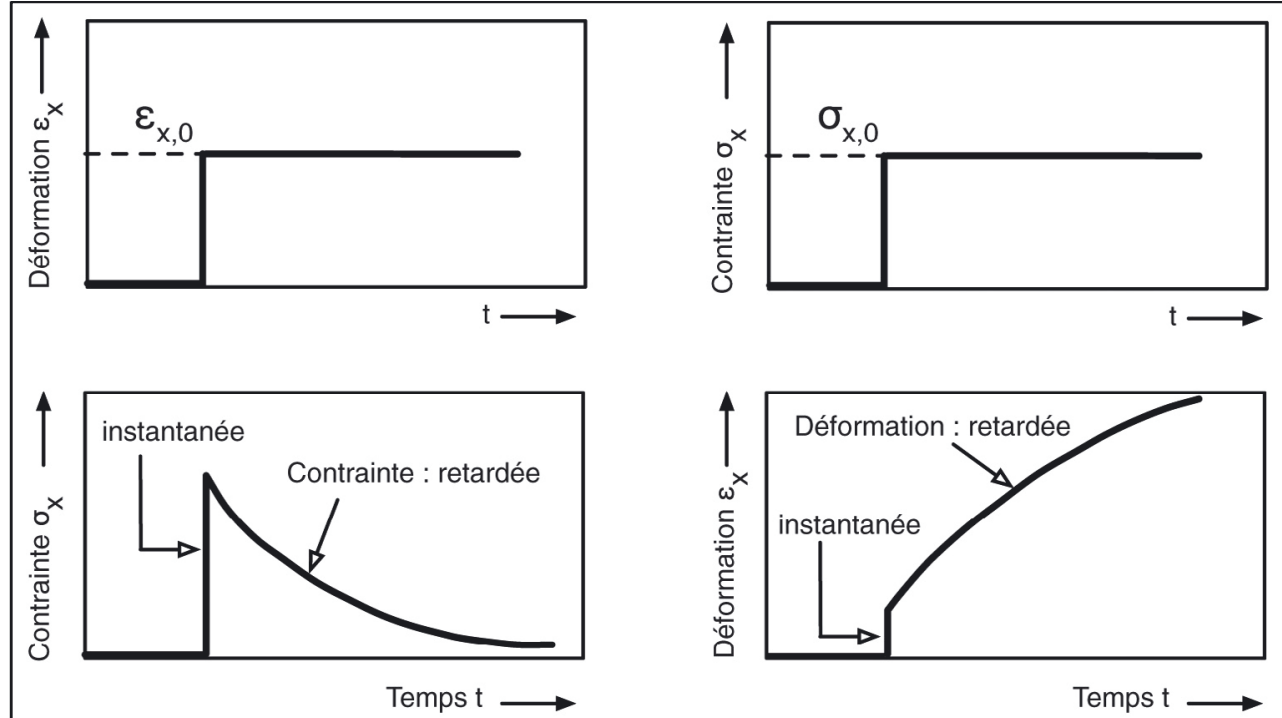

a

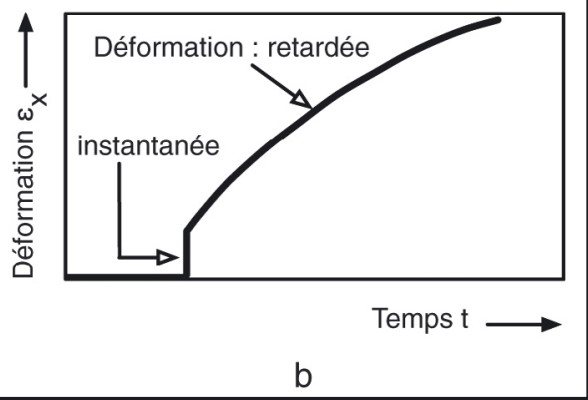

Figure 6

Deux modes de déformation rencontrés en visco-élasticité. (a) : Relaxation des contraintes. (b) : Fluage. (D’après Mercier, et al. [37].)

Huget, et al. (en [11]) ont observé que l'eau agit comme un plastifiant en fragilisant les forces intermoléculaires des polyuréthanes, conduisant à une dégradation chimique. Ces effets délétères seraient dus à la susceptibilité à l'hydrolyse des groupements ester ou ether.
Eliades, et al. [18] ont étudié les modules élastomériques in vivo. Après $24 \mathrm{~h}$ d'exposition, la surface des modules est recouverte d'un film protéique discontinu riche en groupements alcool et avec peu de minéralisation de potassium et de sodium. Après trois semaines, le film protéique devient 


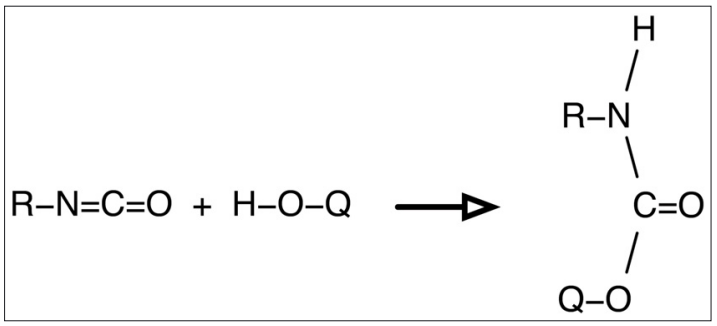

a

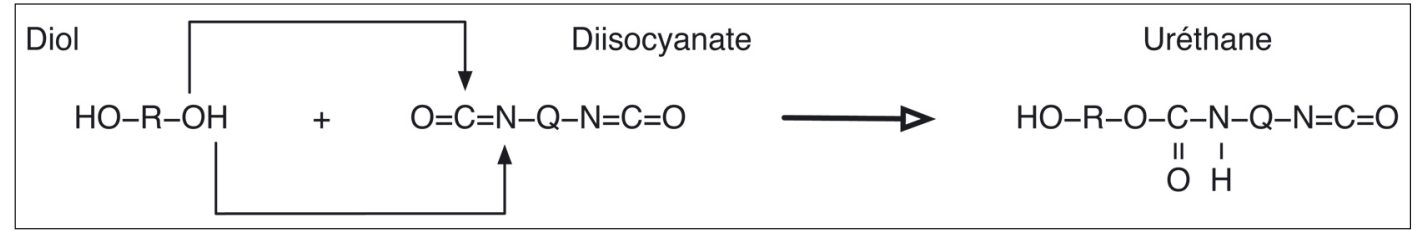

b

Figure 7

Structure générale d'un groupement uréthane $(\mathrm{OCONH})$ formé par réaction d'un diol (a) et d'un diisocyanate (b) (d'après Brantley, Eliades [11]).

très minéralisé, composé de phosphate de calcium avec des impuretés à base de carbonate et d'acide phosphate. Lanalyse aux rayons $\mathrm{X}$ révèle une répartition uniforme de $\mathrm{K}, \mathrm{Na}, \mathrm{Cl}$, et $\mathrm{S}$ pour les numéros atomiques bas, alors que dans les numéros atomiques élevés, on retrouve essentiellement du Ca et du P. Cette absorption de protéines peut ensuite agir comme nucléi et former des dépôts de microcristaux de $\mathrm{Na}, \mathrm{K}$, et $\mathrm{Cl}$, qui peuvent compromettre les performances des modules.

Les variations de $\mathrm{pH}$ et de température dans l'environnement oral, ainsi que l'accumulation de plaque et la formation de colonies bactériennes à la surface des élastomères, peuvent affecter la structure, les propriétés de surface, et la conformation des polyuréthanes. Des élastomères récupérés après peu de temps de port en bouche montrent une surface protéique avec peu de trace de minéralisation du phosphate. Après trois semaines, il y a précipitation des ions $\mathrm{Ca}$ et $\mathrm{P}$, formant des phosphates de calcium à la surface des modules élastomériques. Ces phénomènes sont similaires à ceux observés lorsque des biomatériaux sont exposés à des fluides corporels [11].

\section{Utilisations orthodontiques des élastomères}

Les polyuréthanes constituent le composant principal des matériaux élastomériques en orthodontie.
Les polyuréthanes sont des matériaux dont les performances sont très variées selon les associations chimiques que l'on réalise. On trouvera aussi bien des variétés thermoplastiques que des variétés thermodurcissables ou élastomériques.

Ils peuvent être obtenus par polymérisation, polycondensation ou polyaddition.

Un polyuréthane est un polymère d'uréthane, une molécule organique. On appelle uréthane tout composé produit par la réaction d'un isocyanate et d'un alcool conformément à la réaction suivante illustrée dans les figures $7 \mathrm{a}$ et $7 \mathrm{~b}$.

D’une façon générale, la fonction isocyanate $-\mathrm{N}=\mathrm{C}=\mathrm{O}$ avec deux doubles liaisons présentera une grande réactivité de deux types [1] :

- avec des donneurs d'hydrogène (eau, alcools, amines),

- ou avec un autre isocyanate (dimérisation, trimérisation).

Les segments esters augmentent les propriétés de résistance à l'usure, la capacité de résistance à l'élongation ainsi que la résistance chimique et la stabilité aux UV.

Les segments éthers sont préférés pour accroître la flexibilité à basse température, augmenter la résistance aux attaques microbiennes et décroître les dégradations de type hydrolytique.

Les fournisseurs sont très imprécis quant à la composition des ligatures et chaînettes. De plus, il 
n'est jamais mentionné le nombre de monomère résiduel, surtout lorsque l'on utilise des polymères de diisocyanate (très toxiques).

Composition générale des élastomères [1] :

- Polymère (appelé parfois « résine ») qui confère ses principales propriétés au matériau.

- Charges ou produits de renforcement qui augmentent le module d'élasticité, la ténacité (résistance à la rupture), la résilience (résistance au choc). Les charges sont minérales (craie, talc, mica, amiante) ou organiques (farine de bois, cellulose pure).

- Adjuvants : plastifiants qui rendent le polymère plus flexible et plus huileux au toucher, diminuent la température de transition vitreuse, stabilisants qui évitent la dégradation du polymère sous l'augmentation de la température, colorants, pigments, lubrifiants internes.

\subsection{Ligatures élastomériques}

Leur composant principal est un polyuréthane. Certaines ligatures sont des polyuréthanes avec injection de silicone.

Les ligatures élastomériques sont utilisées pour solidariser l'arc dans la gorge du bracket.

Une étude sur les caractéristiques des ligatures et de la force délivrée [49] montrent :

- une corrélation positive entre épaisseur et force délivrée,

- une corrélation négative entre diamètre interne et force délivrée,

- une corrélation faible entre diamètre externe et force délivrée.

Les ligatures grises délivrent moins de force que les ligatures métalliques $[29,30]$.

\subsubsection{Déperdition de force}

Selon une étude de Taloumis, et al. [49] sur la déperdition de forces des ligatures élastomériques dans un milieu buccal expérimental : la perte est exponentielle et atteint 50-60\% durant les premières $24 \mathrm{~h}$, puis la force continue à diminuer pendant 7 à $10 \mathrm{j}$. Enfin, des signes de déformation permanente et d'altération de la forme deviennent évidents.

L'humidité et la chaleur amplifient la perte de force et la déformation permanente des ligatures élastomériques [49].
Des études in vitro après 12 semaines d'étirement des ligatures élastomériques montrent qu'il existe des différences de déperdition de force entre ligatures claires et colorées et qu'il existe également des différences entre les différentes marques de ligatures [33].

Une étude de 1996 [36] a montré que la désinfection des ligatures et chaînettes entre chaque patient pouvait créer des dégradations et des porosités, donc pouvait endommager la structure de la ligature; sans compter que cela peut engendrer des contaminations croisées. Le mieux est d'utiliser un seul stick de ligature propre à chaque patient.

Une étude sur les effets de la désinfection [22] a montré que le type de désinfectant a un effet sur la température de transition vitreuse : après $1 \mathrm{~h}$ d'exposition à un désinfectant, la force diminue significativement, et la température de transition vitreuse est également affectée, mais de façon différente selon les désinfectants.

\subsubsection{Friction et ligatures élastomériques}

Le principal défaut des ligatures élastomériques est qu'elles engendrent de la friction $[29,30]$. Ceci est un inconvénient majeur dans les techniques de glissement. De nombreuses études ont étudié ce phénomène et ont démontré l'intérêt d'utiliser des ligatures métalliques ou des brackets autoligaturants.

Pour pallier cet inconvénient, les fabricants ont mis sur le marché des ligatures recouvertes. Des études in vitro ont montré que :

- Les modules élastiques recouverts de Metafasix (liaisons covalentes) présentent $50 \%$ de friction en moins par rapport aux autres méthodes de ligature (sauf brackets autoligaturants) [25].

- Les modules élastiques imprégnés de silicone réduiraient de même la friction de $23 \%$ à $43 \%$ par rapport aux ligatures non lubrifiées. La salive n'aurait pas d'effet délétère sur le revêtement [25].

- Les ligatures petites et medium ont une friction réduite de 13-17\% par rapport aux ligatures larges; les ligatures lubrifiées une friction réduite de 23-24\% par rapport aux non-lubrifiées [13].

\subsubsection{Relarguage de fluor}

Les résultats contradictoires obtenus par les différentes études concernant les ligatures élastomériques 
relarguant du fluor sont dus aux variations dans les protocoles expérimentaux des études in vitro [11].

- Certains auteurs comme Wilson ont montré que pendant une période de deux semaines les colonies locales de Streptococcus mutans sont sur le déclin [53] et que la microdureté de l'émail adjacent est augmentée sur $20 \mu \mathrm{m}$ de profondeur [54]. Bien que le taux d'élution a été caractérisé dans une étude comme subissant un fort déclin après trois semaines, Wiltshire a reporté une élution mesurable après six mois in vitro $[55,56]$.

Le problème de la majorité de ces études est qu'elles sont réalisées in vitro, et que la complexité du milieu intrabuccal rend ces résultats peu transposables.

Le relarguage de fluor serait plus important in vivo que in vitro [50].

Des essais cliniques [5,35] ont montré que l'incidence des décalcifications est moins élevée. Une autre étude [15] n’a pas mis en évidence de bénéfice anti-cariogène.

Une étude in vivo n'a pas relevé de différence sur le nombre de colonies de Streptococcus mutans à 7,14 et $28 \mathrm{j}$ [38]; une autre étude a montré qu'elles ne diminuent pas la croissance des bactéries anaérobies et des streptocoques in vivo [8].

- D'autres auteurs comme Storie, et al. [48] ont étudié les effets potentiels sur les propriétés mécaniques. Après une semaine, les élastomères relarguant du fluor ne délivreraient plus une force suffisante, alors que les élastomères classiques délivrent une force requise pendant trois semaines. Létirement augmente la quantité de fluor relâchée [42].

Une étude a même montré qu'en présence de ligatures fluorées la température gingivale augmente de $0,3^{\circ} \mathrm{C}$, ce qui n'a pas de réelle signification clinique [4].

\subsection{Chaînettes élastomériques}

Avec l'essor des techniques d'arc droit, l'utilisation de chaînettes est devenue indispensable pour différentes phases de traitement:

- fermer des espaces,

- maintenir des dents entre elles,

- corriger des rotations,

- recul canin, incisif,

- mésialisation des secteurs postérieurs,
- traction des dents incluses.

Les chaînettes élastomériques existent sous différentes formes:

- chaînettes à modules serrés,

- chaînettes à modules rapprochés,

- chaînettes à modules espacés.

Elles peuvent être transparentes, grises, ou de différentes couleurs.

\subsubsection{Composition}

La composition exacte des chaînettes est gardée secrète par les différents fabricants de matériel orthodontique. Le composant principal est également un polyuréthane.

La polymérisation synthétique ne produit pas, comme la polymérisation naturelle, de longues chaînes moléculaires mais des chaînes plus ou moins longues avec rectifications et chaînes latérales. On cherche à obtenir une polymérisation qui aboutira à la production de chaînes les plus régulières possible. La cohésion moléculaire faible est assurée par des liaisons primaires et secondaires. Les chaînes moléculaires linéaires sont repliées sur elles-mêmes et leur répartition géométrique est aléatoire [32].

\subsubsection{Activation et force délivrée}

Les chaînettes élastomériques doivent avoir une force élastique et un module élastique suffisants pour éviter des ruptures prématurées et fournir les niveaux désirés de force délivrée [11].

L'intensité de la force initiale est très variable en fonction des produits utilisés par les fabricants. La littérature est confuse au sujet des allongements requis pour générer des forces compatibles avec des mouvements dentaires efficaces. Quelques-unes de ces chaînettes, étirées à 100 \% de leur longueur initiale, génèrent des forces d'intensité excessive (450 g), conduisant les chercheurs à recommander un étirement de 50 à 75 \%. D'autres chaînettes, étirées à $100 \%$, donnent des forces acceptables de $300 \mathrm{~g}$. Le praticien doit donc utiliser un dynamomètre [6].

Les chaînettes à modules espacés délivrent des forces initiales plus légères pour un même étirement et subissent, sous l'effet de la tension, une déperdition de force supérieure à celle des chaînettes serrées $[6,14]$. 


\subsubsection{Déperdition de la force délivrée}

Dans l'idéal, la déperdition de force entre deux activations serait minimale et la force générée d'intensité constante pendant cette période. Malheureusement, d'après de nombreuses études, il semble que la déperdition de force ne se fasse pas de manière optimale.

Dans les années soixante-dix, les études sur la relaxation montraient que les chaînettes perdaient en moyenne $50 \%$ de leur force initiale au bout de $24 \mathrm{~h}$ et $70 \%$ au bout de quatre semaines [32].

Selon une revue de la littérature de 1970 à 1994 [6], tous les auteurs s'accordent pour dire que la déperdition de force des chaînettes est plus importante le premier jour, puis elle est ensuite régulière et beaucoup plus faible. Cette déperdition est en moyenne de 40 à $60 \%$ de la force initiale au bout de quatre semaines en fonction des différentes chaînettes et des différentes études.

Dans les années quatre-vingt-dix, il est montré qu'en moyenne les chaînettes perdent environ 30 \% de leur force initiale au bout de 24 h et 50 à $70 \%$ au bout de quatre semaines [32] :

- Étude de relaxation sur les chaînettes «Power chain $\mathrm{Il}^{\circledR}$ » avec une charge de $300 \mathrm{~g}$ : déperdition de force de $36,7 \%$ à 5 min, $53 \%$ en 4 semaines.

- Étude de fluage : en charge de 300 g, la chaînette s'est allongée de $12,7 \%$ à 5 min, 28,8 \% en 24 h, $39,4 \%$ d'allongement en $28 \mathrm{j}$.

- Il existe une relation entre les courbes de relaxation et de fluage.

D’un consensus général, il se produit un déclin de la force de 40 à $50 \%$ durant les premières 24 h, puis la déperdition de force continue à un taux plus faible pendant deux à trois semaines. Ash et Nicolai [2] ont montré que ce phénomène est plus important in vivo que in vitro.

C'est pourquoi certains recommandent d'éviter l'utilisation de chaînettes élastomériques, et d'utiliser des ressorts en $\mathrm{Ni}$ - $\mathrm{Ti}$ avec lesquels la déperdition de force est beaucoup moins importante [46].

Les chaînettes à modules espacés présentent des élongations résiduelles plus importantes que les serrées, ceci serait dû à une élongation importante au niveau des régions intermodulaires, mais les différences varient selon les marques (en raison de la

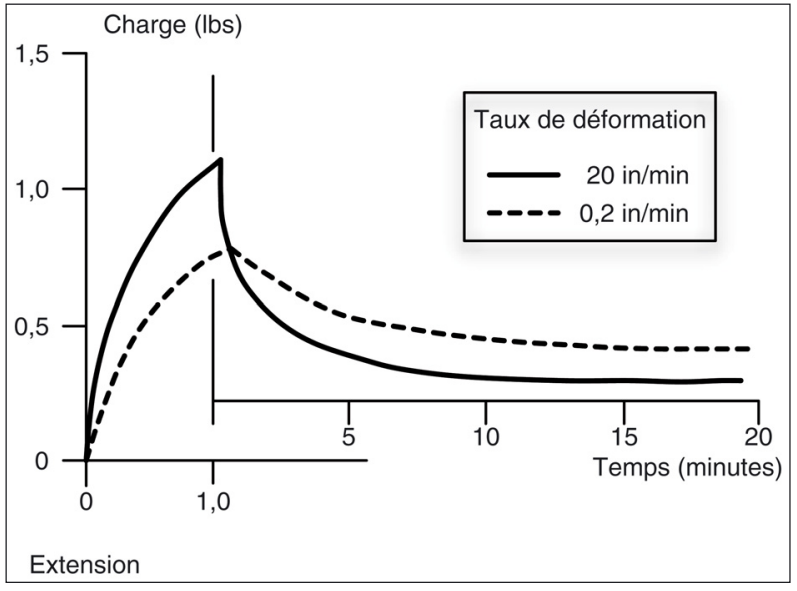

Figure 8

Dépendance entre la vitesse d'extension et la relaxation d'un module élastomérique en polyuréthane (d'après Kovatch, et al. voir [6]).

variabilité de la longueur entre les modules, et la forme des modules) [19].

Plus la chaînette est étirée, plus la force initiale délivrée est importante et plus la force résiduelle sera faible [34].

Le mode de déformation joue également un rôle très important [19] (Fig. 8) :

- Étirement à un rythme lent ou à haute température : la courbe charge-flexion dévie de la ligne droite, le matériau est plus compliant.

- Étirement à un rythme rapide ou à basse température : le matériau se comporte plus comme un corps rigide, car les segments n'ont pas le temps d'être mobilisés et il n'y a pas de glissement entre les chaînes.

Les chaînettes sont très sensibles aux chocs thermiques et aux variations de température [6].

Un phénomène de vieillissement thermique des chaînettes peut exister avant leur utilisation en bouche, d'où l'importance du rôle du milieu de conservation.

La désinfection des chaînettes à l'autoclave entraîne une déperdition de leur force [6].

Cependant, une étude [14] a abouti à la conclusion que les chaînettes élastomériques soumises à un environnement thermique variable (de $15{ }^{\circ} \mathrm{C}$ à $45^{\circ} \mathrm{C}$ ) maintiennent une force résiduelle plus importante que celles soumises à une température constante de $37^{\circ} \mathrm{C}$.

Les chaînettes perdent moins de force dans un milieu basique qu'en milieu acide : ainsi une 


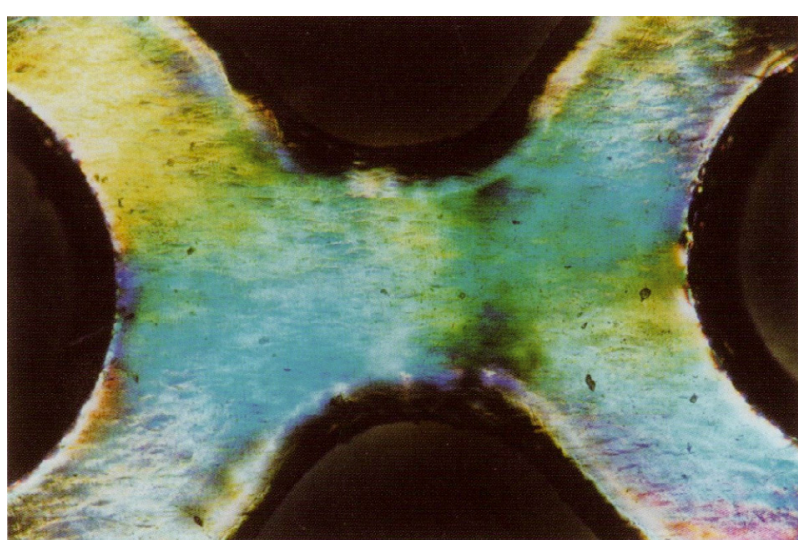

a

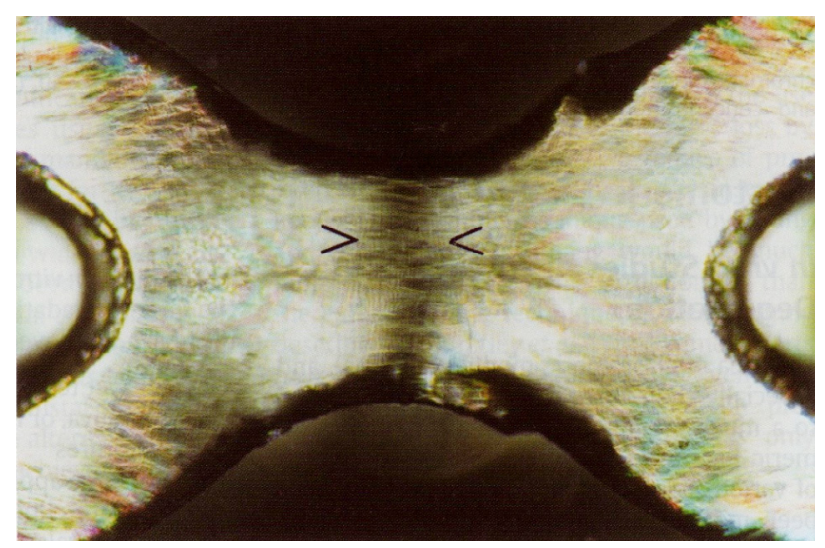

b

Figure 9

Images en lumière polarisée (a) après dépose (b) pour une chaînette étirée à 50 \% pendant 24 h. Noter la direction des franges (zones sombres) au milieu de l'espace intermodulaire et à la liaison avec la zone des anneaux. Grossissement $\times 2,5$. (D'après Brawtley, Eliades [11].)

étude [41] a montré une déperdition de force des chaînettes élastomériques plus importante dans le Cola et le curcuma que dans l'eau.

Par contre, il n'y a pas de différence significative concernant la résistance à la traction entre des chaînettes étirées à l'air et en bouche [19].

\subsubsection{Pré-extension des chaînettes}

Un étirement important crée des microdéchirures (Fig. 9a et 9b) qui partent de la périphérie, et sont perpendiculaires à la direction générale de la châ̂nette [11]. Dans les chaînettes espacées, ces microdéchirures se trouvent entre les oeillettons [21].

Kovatch en 1976 (en [6]) préconisait d'étirer les chaînettes doucement lors de leur mise en place pour augmenter leur efficacité (les chaînettes tendues rapidement délivrent une force majorée, celles étirées plus lentement ont une relaxation minorée).

De 1976 à 1992, le pré-étirement est conseillé par de nombreux auteurs car il permet d'obtenir des forces plus régulières et plus constantes et de réduire la déperdition rapide de leur force [12] :

- pré-étirement dans de l'eau à $37^{\circ} \mathrm{C}$ pendant trois semaines mais pas à l'air,

- pré-extension trop importante qui risque d'engendrer une déformation plastique [32].

Deux modes de pré-étirement ont été proposés [11] :

- technique instantanée proposée par Sandrik et Chang,
- pré-étirement durant une longue durée par Brantley.

Le pré-étirement diminuerait seulement de $10 \%$ de la perte de force [6].

Par contre, selon Kim [31], en 2005, l'effet de la pré-extension n'est significatif que pendant la première heure.

Les chaînettes introduisent dans l'exercice de l'orthodontie une imprécision quant aux forces exercées sur les structures dentaires. Pour chaque type de chaînette, les fabricants devraient indiquer la force exercée en fonction de l'allongement et la déperdition en fonction du temps. Employer des matériaux possédant un domaine d'élasticité pur, comme des ressorts métalliques [32] permettrait de connaître avec précision les forces appliquées.

Des perspectives d'amélioration sont attendues pour ces matériaux [17] :

- polymères avec relaxation réduite et prédéterminée,

- emploi de polymères qui relarguent du fluor mais avec une relaxation améliorée,

- élastomères recouverts d'un film pour diminuer la réactivité, l'absorption d'eau et la dégradation.

\subsection{Les élastiques}

Depuis le début des années quatre-vingt-dix, des élastiques synthétiques sont apparus sur le marché pour les patients présentant une allergie au latex [27]. 
La rigidité de l'élastique dépend de sa nature mais aussi de son épaisseur : plus il est épais, plus il est rigide.

Pour obtenir la force requise, il faut étirer entre 2,7 à 5 fois la longueur initiale de l'élastique. La règle empirique des «trois fois » n'est pas toujours valable [24].

Les élastiques présentent également une perte de force très importante durant les trois à cinq premières heures puis qui se stabilise [24], quels que soient la taille, le fabricant, la force délivrée. Le maximum de perte serait au cours de la première demiheure [52].

Les élastiques plus fins ont une déperdition de force plus importante [7].

La relaxation moyenne des élastiques en latex serait de $25 \%$ [24]. Les élastiques en latex ont une perte de force plus importante in vivo qu'in vitro; et plus importante en traction intermaxillaire qu'en traction intramaxillaire [52].

Les élastiques sans latex ont une perte de force significativement plus importante après $24 \mathrm{~h}(50 \%$ au bout de $24 \mathrm{~h}$ [28]) et sont plus déformables $[9,27]$; c'est pourquoi ils doivent être changés plus souvent que ceux avec latex [23]

Les différences de relaxation entre élastiques en latex et sans latex sont dues au matériau. La force de rétraction délivrée par des élastiques synthétiques provient de l'enchevêtrement des chaînes. À l'inverse, les caoutchoucs naturels tels le latex délivrent une force due à des liaisons covalentes et à des ponts croisés [24]. Il a également été émis l'hypothèse que différents facteurs environnementaux, comme l'humidité et la chaleur, pourraient agir différemment et probablement avoir plus d'effets néfastes sur les élastiques sans latex en comparaison avec ceux en latex, en rapport avec leurs différences de structure [27].

Lalimentation n'aurait pas ou peu d'influence comme l'a prouvé l'étude de Beattie, et al. en 2007 [7] : différents régimes alimentaires n'ont pas d'influence; les élastiques en latex maintiennent la force délivrée après un jour de port.

Une augmentation de la température de l'environnement a une influence significative sur le mécanisme de dégradation responsable de la détérioration des propriétés mécaniques des élastiques à base de polyuréthane, alors que l'acidité et le contenu en oxygène n'ont pas ou peu d'action [47].

\section{4. Élastodontie}

En 1945, Kesling a introduit le «tooth positionner », appareil utilisé pour la finition des traitements orthodontiques. Depuis, différents matériaux ont été utilisés dont un caoutchouc polyuréthane. Actuellement est souvent employé un "positionneur » en silicone à basse température. Yoshii à Tokyo a développé en 1980 le concept de «positionneur dynamique », et a publié des cas cliniques réalisés du début de traitement à la contention en deux ou trois « positionneurs ». Il utilisait pour cela un silicone à haute température de vulcanisation, l'Orthocon, en trois types d'élasticité. Ce concept est alors devenu l'élastodontie et l'appareil original de Yoshii a été appelé l'Osamu.

Les dents sont déplacées en utilisant l'élasticité d'un matériau élastomérique permettant de fabriquer une gouttière bimaxillaire à partir d'une maquette prévisionnelle (set-up) intégrant les objectifs de traitement et le schéma occlusal recherché. Le matériau élastomérique existe dans trois duretés différentes:

- dure, pour les zones d'ancrage;

- moyenne, pour les déplacements de petite amplitude et la correction des rotations;

- souple, pour les mouvements d'ingression, d'égression, les déplacements importants et la fermeture des diastèmes.

Trois types d'appareils sont actuellement décrits :

- Les élasto-Osamu 1 et 2 sont des appareils à vocation fonctionnelle. On peut y adjoindre une force extra-buccale. Une «cire de construction » règle le degré de propulsion mandibulaire souhaité et un arc interne d'expansion est incorporé à la gouttière.

- L'elasto-aligneur permet d'aligner les dents. Des attaches sont souvent collées, surtout si des mouvements de troisième ordre sont recherchés.

- L'elasto-finisseur est un appareil d'idéalisation des arcades, de finition précise de l'intercuspidation [45].

\section{Conclusion}

De nombreuses études ont montré des différences de relaxation des matériaux élastomériques 
selon les fabricants. On pourrait retenir différentes causes $[11,20]$ :

- technique de fabrication (découpe ou moulage par injection à partir du matériau à l'état brut), mais une étude [10] n'a pas mis en évidence de différence significative de la force résiduelle après trois semaines in vivo;

- effets induits par différents additifs incorporés dans le produit final ;

- rigueur des contrôles qualité;

- forme ellipsoïde ou circulaire des modules;

- diamètre des modules.

De plus, les différentes études in vitro ont des paramètres qui diffèrent, les rendant ainsi difficilement comparables :

- tests en milieu sec ou humide : eau, salive artificielle, fluor, et à différentes températures;

- forces constantes ou forces décroissantes pour simuler les conditions cliniques;

- pH acide ou neutre.

\section{Bibliographie}

[1] Amar-Mamou I. Comportement colorimétrique des auxiliaires élastomériques esthétiques en fonction du temps en orthodontie (étude in vitro). Mémoire CECSMO. Paris: Université Diderot, 2004.

[2] Ash JL, Nikolai RJ. Relaxation of orthodontic elastomeric chains and modules in vitro and in vivo. J Dent Res, 1978;57:685-690.

[3] Ashby MF, Jones DRH. Matériaux. Tome 1 : Microstructure et mise en œuvre. Tome 2 : Propriétés et applications. Paris: Ed. Dunod, 1998.

[4] Aznan K, Khan M, Benson PE. Gingival temperature measurements with fluoride and nonfluoride elastomeric ligatures. Am J Orthod Dentofacial Orthop 2007;131:378-383.

[5] Banks PA, Chadwick SM, Asher-McDade C, Wright JL. Fluoride-releasing elastomerics-a prospective controlled clinical trial. Eur J Orthod 2000;22:401-407.

[6] Baty DL, Storie DJ, Von Fraunhofer JA. Synthetic elastomeric chains: a literature review. Am J Orthod Dentofacial Orthop 1994;105:536-542

[7] Beattie S, Monaghan P. An in vitro study simulating effects of daily diet and patient elastic band change compliance on orthodontic latex elastics. Angle Orthod 2004;74:234-239.

[8] Benson PE, Douglas CW, Martin MV. Fluoridated elastomers: effect on the microbiology of plaque. Am J Orthod Dentofacial Orthop 2004;126:325-330.
[9] Bertoncini C, Cioni E, Grampi B, Gandini P. In vitro properties' changes of latex and non-latex orthodontic elastics. Prog Orthod 2006;7:76-84.

[10] Bousquet JA Jr, Tuesta O, Flores-Mir C. In vivo comparison of force decay between injection molded and diecut stamped elastomers. Am J Orthod Dentofacial Orthop 2006;129:384-389.

[11] Brantley WA, Eliades T. Orthodontic Materials. Scientific and Clinical Aspects. Stuttgart, New York: Thieme, 2001, $310 \mathrm{p}$.

[12] Chassaing-Wolton I. Mesures des variations des forces délivrées par les chaînettes élastomériques en fonction de leurs altérations dans le temps (étude in vitro et in vivo). Mémoire CECSMO. Paris: Université D. Diderot, 1996.

[13] Chimenti C, Franchi L, Di Giuseppe MG, Lucci M. Friction of orthodontic elastomeric ligatures with different dimensions. Angle Orthod 2005;75:421-425.

[14] De Genova DC, McInnes-Ledoux P, Weinberg R, Shaye R. Force degradation of orthodontic elastomeric chains - a product comparison study. Am J Orthod 1985;87:377-384.

[15] Doherty, UB, Benson PE, Higham SM. Fluoride-releasing elastomeric ligatures assessed with the in situ caries model. Eur J Orthod 2002;24:371-378.

[16] Ebaïlon JP, Dorlot JM. Des matériaux. $3^{e}$ édition. Paris: Ed. Presses Internationales Polytechnique, 2000, 736 p.

[17] Eliades T. Orthodontic materials research and applications: part 2. Current status and projected future developments in materials and biocompatibility. Am J Orthod Dentofacial Orthop 2007;131:253-262.

[18] Eliades T, Bourauel C. Intraoral aging of orthodontic materials: the picture we miss and its clinical relevance. Am J Orthod Dentofacial Orthop 2005;127:403-412

[19] Eliades T, Eliades G, Silikas N, Watts DC. Tensile properties of orthodontic elastomeric chains. Eur J Orthod 2004;26:157-162.

[20] Eliades T, Eliades G, Silikas N, Watts DC. In vitro degradation of polyurethane orthodontic elastomeric modules. J Oral Rehabil 2005;32:72-77.

[21] Eliades T, Eliades G, Watts DC. Structural conformation of in vitro and in vivo aged orthodontic elastomeric modules. Eur J Orthod 1999;21:649-658.

[22] Evangelista MB, Berzins DW, Monaghan P. Effect of disinfecting solutions on the mechanical properties of orthodontic elastomeric ligatures. Angle Orthod 2007;77:681-687.

[23] Gandini P, Gennai R, Bertoncini C, Massironi S. Experimental evaluation of latex-free orthodontic elastics' behaviour in dynamics. Prog Orthod 2007;8:88-99.

[24] Gioka C, Zinelis, S, Eliades T, Eliades G. Orthodontic latex elastics: a force relaxation study. Angle Orthod 2006;76:475-479.

[25] Hain M, Dhopatkar A, Rock P. A comparison of different ligation methods on friction. Am J Orthod Dentofacial Orthop 2006;130:666-670. 
[26] Huet A, Montandreau E. Étude des chaînettes élastomériques et de leur relaxation. Orthod Fr 1993;64:619-633.

[27] Kersey ML, Glover KE, Heo G, Raboud D, Major PW. A comparison of dynamic and static testing of latex and nonlatex orthodontic elastics. Angle Orthod 2003; $73: 181-186$

[28] Kersey ML, Glover K, Heo G, Raboud D, Major PW. An in vitro comparison of four brands of nonlatex orthodontic elastics. Am J Orthod Dentofacial Orthop 2003;123:401-407.

[29] Khambay B, Millett D, McHugh S. Evaluation of methods of archwire ligation on frictional resistance. Eur J Orthod 2004:26:327-332

[30] Khambay B, Millett D, McHugh S. Archwire seating forces produced by different ligation methods and their effect on frictional resistance. Eur J Orthod 2005:27:302-308.

[31] Kim KH, Chung CH, Choy K, Lee JS, Vanarsdall RL. Effects of prestretching on force degradation of synthetic elastomeric chains. Am J Orthod Dentofacial Orthop 2005; 128:477-482

[32] Lagache S. Etude in vitro du fluage et de la relaxation des chaînettes élastomériques en Orthodontie. Mémoire CECSMO. Lille: Université de Lille 2, 2006.

[33] Lam TV, Freer TJ, Brockhurst PJ, Podlich HM. Strength decay of orthodontic elastomeric ligatures. J Orthod 2002;29:7-43.

[34] Lu TC, Wang WN, Tarng TH, Chen JW. Force decay of elastomeric chain - a serial study. Part II. Am J Orthod Dentofacial Orthop 1993;104:373-377.

[35] Mattick CR, Mitchell L, Chadwick SM, Wright J. Fluoridereleasing elastomeric modules reduce decalcification: a randomized controlled trial. J Orthod 2001;28:217-219.

[36] Mayberry D, Dallen R, Close J, Kinney DA. Effects of desinfection procedures on elastomeric ligatures. J Clin Orthod 1996;30:49-51.

[37] Mercier JP, Zambelli G, Kurz W. Introduction à la science des matériaux. Lausanne: Presses Polytechniques et Universitaires romandes, 1999.

[38] Miura KK, Ito IY, Enoki C, Elias AM, Matsumoto MA. Anticariogenic effect of fluoride-releasing elastomers in orthodontic patients. Braz Oral Res 2007;21:228-233.

[39] Montandreau E. Études théoriques et expérimentales sur les chaînettes élastomériques. Mémoire CECSMO. Lyon: Université Claude Bernard - Lyon 1, 1992.

[40] Mousseau J. À propos des forces élastiques en orthodontie. Thèse Doct Etat. Nantes: Université de Nantes, 1996.

[41] Nattrass C, Ireland AJ, Sherriff M. The effect of environmental factors on elastomeric chain and nickel titanium coil springs. Eur J Orthod 1998;20:169-176.
[42] O'Dwyer JJ, Tinsley D, Benson PE. The effect of stretching on the release of fluoride from fluoridated elastomeric ligatures. Am J Orthod Dentofacial Orthop 2005;128:471-476.

[43] Philippe J. Qui a inventé... les élastiques d'orthodontie ? Rev Orthop Dento Faciale 2001;35:145-150.

[44] Renick MR, Brantley WA, Beck FM, Vig KW, Webb CS. Studies of orthodontic elastomeric modules. Part 1: Glass transition temperatures for representative pigmented products in the as-received condition and after orthodontic use. Am J Orthod Dentofacial Orthop 2004;126:337-343

[45] Rollet D, Graindorge JC, Guezennec P. Un concept nouveau : l'élastodontie. Rev Orthop Dento Faciale 1991;25:149-167.

[46] Santos AC, Tortamano A, Naccarato SR, DominguezRodriguez GC, Vigorito JW. An in vitro comparison of the force decay generated by different commercially available elastomeric chains and Ni-Ti closed coil springs. Braz Oral Res, 2007;21:51-57.

[47] Stevenson JS, Kusy RP. Force application and decay characteristics of untreated and treated polyurethane elastomeric chains. Angle Orthod 1994;64:455-464.

[48] Storie DJ, Regennitter F, Von Fraunhofer JA. Characteristics of a fluoride-releasing elastomeric chain. Angle Orthod 1994;64:199-209

[49] Taloumis LJ, Smith TM, Hondrum SO, Lorton L. Force decay and deformation of orthodontic elastomeric ligatures. Am J Orthod Dentofacial Orthop 1997;111:1-11.

[50] Tinsley D, O'Dwyer JJ, Benson PE. Fluoridated elastomers: in vivo versus in vitro fluoride release. J Orthod 2003;30: 317-322.

[51] Trotignon JP, Verdu J, Dobraczynski A, Pieraud M. Précis de matières plastiques. Structures, propriétés, mise en œuvre, normalisation. Paris: Nathan, 1991.

[52] Wang T, Zhou G, Tan X, Dong Y. Evaluation of force degradation characteristics of orthodontic latex elastics in vitro and in vivo. Angle Orthod 2007;77:688-693.

[53] Wilson TG, Gregory RL. Clinical effectiveness of fluoride-releasing elastomers. I: Salivary Streptococcus mutans numbers. Am J Orthod Dentofacial Orthop $1995 ; 107: 293-297$

[54] Wilson TG, Love B. Clinical effectiveness of fluoridereleasing elastomers. II. Enamel microhardness levels. Am J Orthod Dentofacial Orthop 1995;107:379-381.

[55] Wiltshire WA. Determination of fluoride from fluoridereleasing elastomeric ligature ties. Am J Orthod Dentofacial Orthop 1996;110:383-387.

[56] Wiltshire WA. In vitro and in vivo fluoride release from orthodontic elastomeric ligature ties. Am J Orthod Dentofacial Orthop 1999;115:288-292. 